\title{
Skin blood flow response signal using time and frequency domain features for pressure ulcer evaluation
}

\begin{abstract}
Pressure Ulcer (PU) is an area of the skin in which cutaneous tissue is compromised and there is progressive damage on the underlying tissue caused by blood flow obstruction due to prolonged external direct pressure. Research has shown that ischemic stress response can be evaluated using skin blood flow response (SBFR) signal features which are useful for pressure ulcer evaluation. Trends of peak reactive hyperemia (RH) were observed for three repetitive loading-unloading cycles in previous animal study to investigate tissue recovery. However, tissue recovery and tissue damage cannot be discriminated by the trends of peak RH for short recovery time. The trends of alternative time-domain SBFR features such i.e total hyperemic response as well as frequency-domain features using Fast Fourier Transform (FFT) and Short Time Fourier Transform (STFT) i.e total power spectrum are further investigated to indicate tissue recovery. The results show that total hyperaemic response outperforms peak $\mathrm{RH}$ at detecting insufficient tissue recovery with $72 \%$ of samples with increasing trend in the short recovery time group compared to $57 \%$ of samples for peak $\mathrm{RH}$. Total hyperemic response is effective at discriminating insufficient recovery time while other investigated features are only effective at detecting sufficient recovery time.
\end{abstract}

Keyword: Pressure ulcer; Skin blood flow response; Reactive hyperemia; Time domain features; Frequency domain features; Peak of power spectrum; Total of power spectrum 